\title{
Swirling Flow and Wall Shear: Evaluating the BioMimics 3D Helical Centerline Stent for the Femoropopliteal Segment
}

\author{
Timothy M. Sullivan (D), ${ }^{1}$ Thomas Zeller, ${ }^{2}$ Masato Nakamura, ${ }^{3}$ \\ Colin G. Caro, ${ }^{4}$ and Michael Lichtenberg ${ }^{5}$ \\ ${ }^{1}$ Vascular/Endovascular Surgery, Minneapolis Heart Institute at Abbott Northwestern Hospital, Minneapolis, MN, USA \\ ${ }^{2}$ Department of Angiology, Universitäts-Herzzentrum Freiburg Bad Krozingen, Bad Krozingen, Germany \\ ${ }^{3}$ Division of Cardiovascular Medicine, Toho University, Ohashi Medical Center, Tokyo, Japan \\ ${ }^{4}$ Department of Bioengineering, Imperial College, London, UK \\ ${ }^{5}$ Vascular Center Klinikum Arnsberg, Arnsberg, Germany
}

Correspondence should be addressed to Timothy M. Sullivan; timothy.sullivan@allina.com

Received 11 November 2017; Accepted 31 December 2017; Published 26 February 2018

Academic Editor: Mark Morasch

Copyright (C) 2018 Timothy M. Sullivan et al. This is an open access article distributed under the Creative Commons Attribution License, which permits unrestricted use, distribution, and reproduction in any medium, provided the original work is properly cited.

\begin{abstract}
The BioMimics 3D self-expanding nitinol stent represents a strategy for femoropopliteal intervention that is alternative or complementary to deployment of drug-coated stents or balloons. Whereas conventional straight stents reduce arterial curvature and disturb blood flow, creating areas of low wall shear, where neointimal hyperplasia predominantly develops, the helical centerline geometry of the BioMimics 3D maintains or imparts arterial curvature, promotes laminar swirling blood flow, and elevates wall shear to protect against atherosclerosis and restenosis. In the multicenter randomized MIMICS trial, treatment of femoropopliteal disease with the BioMimics 3D $(n=50)$ significantly improved 2-year primary patency (log-rank test $p=0.05)$ versus a control straight stent $(n=26)$, with no cases of clinically driven target lesion revascularization between 12 and 24 months (log-rank test $p=0.03$ versus controls). In geometric X-ray analysis, the BioMimics stent was significantly more effective in imparting a helical shape even when the arterial segment was moderately to severely calcified. Computational fluid dynamics analysis showed that average wall shear was significantly higher with the helical centerline stent $(1.13 \pm 0.13 \mathrm{~Pa}$ versus $1.06 \pm 0.12 \mathrm{~Pa}, p=0.05)$. A 271-patient multicenter international MIMICS-2 trial and a 500-patient real-world MIMICS-3D registry are underway.
\end{abstract}

\section{Introduction}

Peripheral artery disease (PAD) is one of the most prevalent, morbid, and mortal diseases worldwide, affecting more than 202 million individuals [1]. Between 2000 and 2010, the prevalence of PAD grew at a rate of $13.1 \%$ in high-income countries and $28.7 \%$ in low- and middle-income countries [1]. Patients with PAD have an increased risk of myocardial infarction, stroke, and death, as well as significant quality of life (QOL) impairment. Initial treatment of PAD in patients with intermittent claudication is directed at lifestyle and behavior modification along with medical management in order to slow the disease and symptom progression. When these methods fail to provide symptomatic relief, revascularization is appropriate by endovascular or surgical means, as recommended by current society guidelines [2].

The femoropopliteal arterial segment is the most common anatomic location of occlusive PAD [3]. The superficial femoral artery (SFA) descends along the anteromedial part of the thigh in the femoral triangle before entering and passing through the adductor canal and becoming the popliteal artery, which runs through the knee in close proximity to the joint capsule. The femoropopliteal segment is thus uniquely subjected throughout its length to complex external mechanical stresses, including flexion, compression, and torsion, which may contribute to poor treatment outcomes 


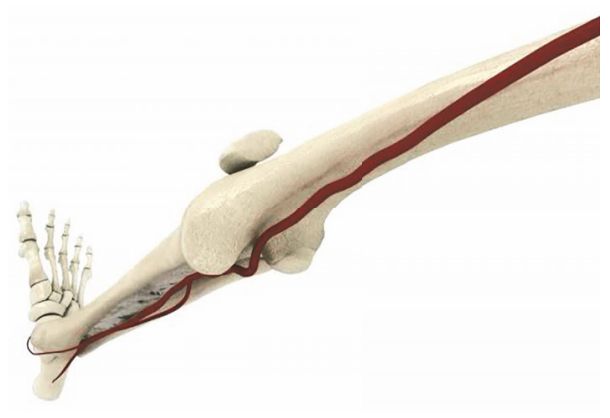

(a)

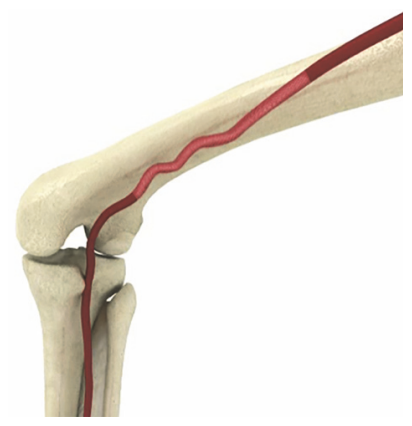

(b)

FIGURE 1: (a) Gentle curvature of the superficial femoral artery (SFA) with the leg extended. (b) With the knee flexed, the distal SFA adopts a helical pattern to accommodate vessel slack.

such as mechanical failure of stents and arterial kinking [3-6]. Atherosclerotic occlusive lesions in the femoropopliteal segment, due to associated blood flow patterns and disturbances and consequent areas of low wall shear, can be long and can involve significant calcification and/or fibrosis [7-9]. The clinical presentation of patients with femoropopliteal lesions can range from asymptomatic or with minor symptoms to QOL-limiting intermittent claudication (IC) or critical limb ischemia (CLI). While, with the leg extended, the SFA has a gentle open spiral shape, during thigh contraction and knee flexion, compression is increased as the distal SFA segment traverses the adductor canal and adopts a more helical pattern (Figure 1).

As endovascular procedures entail lower periprocedural risks and decreased initial costs in comparison with open surgical repair in PAD patients with multiple comorbidities [10], current guidelines recommend an initial endovascular approach for treatment of most types of femoropopliteal disease [11]. The objective in endovascular treatment of PAD is the restoration of patency and blood flow in stenosed or occluded arterial segments, in such a way that restenosis or reocclusion can be avoided along with the need for reinterventions to correct for the failure of primary patency. When primary patency fails, PAD symptoms often recur, providing clinical indications for target lesion revascularization (TLR) or target vessel revascularization (TVR) procedures, which are costly, clinically more risky, and less likely to be successful [12-14]. The causes of low patency rates following endovascular repair of femoropopliteal lesions can include immediate elastic recoil of the treated segment; intimal dissection; late negative vessel remodeling (fibrosis of the adventitia); and an inflammatory process following balloon barotrauma and stent implantation leading to development of neointimal hyperplasia (NIH) and in turn to restenosis [4]. Elastic recoil and intimal dissection can be managed relatively successfully by prolonged balloon dilatation and/or mechanical scaffolding with stents $[4,6]$. With the unsatisfactory outcomes following standalone percutaneous transluminal angioplasty (PTA) in many lesion types (1-year vessel patency $<30 \%$ [15]) and use of nondedicated bare-metal stents [16, 17], the development of further strategies is ongoing to effectively prevent or limit the activation of the NIH cascade and consequent vessel renarrowing.

The specific endovascular options that have been successively developed in the effort to prevent restenosis in the femoropopliteal segment include dedicated self-expanding nitinol stents, drug-eluting stents (DES), and drug-coated balloons (DCBs). In a recent analysis of femoropopliteal outcomes in clinical trials of different endovascular modalities, updated from the systematic review presented in the Cardiovascular and Interventional Radiological Society of Europe (CIRSE) Standards of Practice guideline on the superficial femoral and popliteal arteries, rates of technical success were uniformly high for endovascular treatment of stenoses (ranges between 98\% and 100\%) and occlusions (ranges between $81 \%$ and $94 \%$ ). However, 1-year rates of primary patency ranged from $57 \%$ (95\% confidence interval [CI]: $42 \%-72 \%$ ) for PTA, to $66 \%$ (95\% CI: $67 \%-91 \%)$ for conventional nitinol stents, to $81 \%$ (95\% CI: 67\%-91\%) for DCBs and $83 \%$ (95\% CI: $77 \%-90 \%)$ for DES $[4,18]$. The extent to which these systematic review findings represent treatment of real-world patients is not clear, as the 1-year patency rates were not discriminated on the basis of symptoms (IC versus CLI), comorbidities such as renal disease and diabetes, or lesion characteristics (baseline lesion length, percentage of stenosis, or presence and degree of calcification) [18]. Clinical trials generally enroll patients with shorter rather than longer lesions (lesion lengths ranged from 5 to only $10 \mathrm{~cm}$ in the DES trials represented in the systematic review [4]), fewer rather than more occluded lesions, and only mildly to moderately calcified lesions. Clinical trials likewise tend to exclude patients with Rutherford class 5 and 6 disease (the most severe clinical presentations), and they do not generally involve head-to-head comparisons of related interventional modalities.

In real-world practice, for example, when DCBs are employed as primary treatment of femoropopliteal disease, complementary stent deployment is planned or at least reserved for treatment of suboptimal results. The pivotal DCB trials focused on a closely defined set of lesions arising from relatively uncomplicated $\mathrm{PAD}$ that would not require complementary stenting; they explicitly excluded severe calcification 
and an inability to completely predilate the lesion; and the use of SFA stents was not permitted [19-23]. Consequently, the rates of bailout stenting were only between $2.5 \%$ and $7.0 \%$ in the pivotal DCB trials, whereas the rates of complementary stenting were between $28.8 \%$ and $35.5 \%$ (with the rate of stenting related to lesion length and degree of occlusion) when use of the same DCBs was analyzed in registry studies. In the IN.PACT Global registry, the rate of complementary stenting was $53 \%$ when lesion length exceeded $25 \mathrm{~cm}$. The pharmacokinetic profile of DCBs, while varying with the type of excipient coating employed, is distinct from that of DES in terms of drug-release and tissue-distribution characteristics but still also time limited (with the drug eluted by DCBs remaining at therapeutic levels for less than 1 year), whereas loss of patency in the SFA due to restenosis can occur as late as 3 to 4 years after treatment [24]. That reality suggests a role for complementary deployment of a stent associated with durable outcomes in cases when DCBs are employed as primary treatments.

\section{The BioMimics 3D Helical Centerline Stent}

An alternative or complementary strategy for limiting the $\mathrm{NIH}$ cascade and restenosis associated with endovascular interventions in the femoropopliteal segment focuses on imparting a helical shape to the stented arterial segment and thereby inducing a laminar swirling flow of blood that increases mixing within the blood and generates an antirestenotic and atheroprotective elevation in wall shear. That is the strategy behind development of the BioMimics 3D helical centerline stent system (Veryan Medical, Horsham, UK). The design of the BioMimics 3D builds on the principles underlying the most recent generation of nitinol stents dedicated for use in the femoropopliteal arterial segment-radial support, flexibility, durability, clarity of visualization, and accuracy of delivery-and adds three-dimensional helical centerline geometry, for the purpose of providing biomechanical stability and also generating swirling flow within the stented segment.

This self-expanding nitinol stent is under evaluation in 3 separate multicenter prospective clinical trials in the MIMICS Clinical Trial Program (Table 1). (1) The multicenter MIMICS study, the first-ever randomized trial comparing two differently designed bare-metal nitinol stents (in most nitinol stent studies, the investigational device is compared with PTA) [25]; (2) the prospective MIMICS-2 investigational device exemption (IDE) study, which is now evaluating the helical centerline stent in 271 patients at 43 sites in 3 different countries (the United States, Japan, and Germany); and (3) the prospective observational MIMICS-3D registry, which is evaluating the helical centerline stent in a realworld clinical population, with targeted enrollment of more than 500 patients and with a dedicated subgroup analysis of device performance as a complementary treatment in procedures involving DCBs are included. Clinical results of the randomized MIMICS study, which have been published out to 24 months, demonstrate that differences in stent design do influence clinical outcomes in the femoropopliteal segment [25].
The current review considers the evidence supporting the use of the BioMimics 3D helical centerline stent as an effective alternative or complementary strategy for limiting NIH in the femoropopliteal segment. To contextualize this strategy, the review begins with a summary of what is known about the relationship between wall shear and restenosis.

2.1. Wall Shear, Restenosis, and the Effect of Swirling Flow. It has long been understood that disturbances in arterial fluid mechanics contribute to atherogenesis [26]. Arterial geometry is commonly helical, causing the blood to adopt a laminar swirling flow pattern, with the higher velocity of flow occurring toward the arterial wall rather than in the center of the vessel as in the case of arteries that are straight [27]. The naturally occurring laminar swirling flow increases mixing within the blood, elevates the wall shear on endothelial cells, and promotes the diffusion of oxygen to the arterial wall, protecting against the development of atherosclerosis and restenosis [28, 29]. The vessel endothelium is not a passive nonthrombogenic surface but rather a dynamically responsive vascular element, which produces autocrine and paracrine factors under the functional regulation of local hemodynamic forces [30]. A critical determinant of endothelial function and phenotype [30-32], wall shear is the tangential component of frictional force generated at the vessel wall by the flow of blood. High (normal or physiological) wall shear induces endothelial-cell (EC) quiescence and an atheroprotective gene-expression profile, whereas low shear stress stimulates an atherogenic phenotype (Figure 2) [30]. That is to say that atherosclerosis and NIH mainly occur at locations where wall shear is low $[7,8,26]$-wall shear $>1.5 \mathrm{~Pa}$ being atheroprotective, while wall shear $<0.5 \mathrm{~Pa}$ is related to the development of atherosclerosis and restenosis.

As the SFA is a long, relatively straight vessel, it is exposed to low wall shear under resting conditions [9] - a circumstance that predisposes it to atherosclerotic disease while also serving as a factor that confounds the healing process after endovascular injury. Implantation into the SFA of a stent with a straight cylindrical configuration will act to further straighten the vessel, so that while the stent may restore vessel patency, it can alter effective arterial flexibility and reduce the capability of the vessel to shorten naturally (as in an unstented state), opening the way for kinking and buckling of the vessel at the ends of the stented segment as well as for possible stent fracture [5]. The introduction of a straight stent may jeopardize the antirestenotic swirling blood flow commonly imparted by the natural helical arterial geometry. The implantation of a stent with a helical centerline can avoid this possibility by imparting swirling flow to the diseased segment of the SFA requiring intervention.

2.2. Device Design. The BioMimics 3D helical centerline stent was designed to impart a helical shape to vessel morphology and thereby induce a laminar swirling flow that will elevate antirestenotic and atheroprotective wall shear (Figure 3 ). The strut pattern of the helical centerline stent promotes high device flexibility while still retaining sufficient stiffness to impart a helical shape to the stented segment even when 
TABLE 1: The MIMICS Clinical Trial Program.

\begin{tabular}{|c|c|c|c|}
\hline Trial & MIMICS (NCT02163863) & MIMICS-2 (NCT02400905) & MIMICS 3D (NCT02900924) \\
\hline Structure & Randomized controlled trial & Prospective registry, IDE study & $\begin{array}{l}\text { Prospective registry, postmarket } \\
\text { surveillance }\end{array}$ \\
\hline Enrollment & $\begin{array}{l}\text { Helical centerline arm }(n=50) \\
\text { Control stent } \operatorname{arm}(n=26)\end{array}$ & 271 patients & Up to 500 real-world patients \\
\hline Sites/location & 8 sites/Germany & $\begin{array}{l}35 \text { sites/United States } \\
6 \text { sites/German } \\
6 \text { sites/Japan } \\
\end{array}$ & 25 sites/Europe \\
\hline Lesion type & $\begin{array}{l}\text { Stenotic or occlusive lesions in the } \\
\text { SFA; restenotic lesions permitted }\end{array}$ & $\begin{array}{l}\text { Stenotic or occlusive lesions in the } \\
\text { femoropopliteal artery }\end{array}$ & $\begin{array}{l}\text { Stenotic or occlusive lesions in the } \\
\text { femoropopliteal artery }\end{array}$ \\
\hline $\begin{array}{l}\text { Adjudication } \\
\text { structure }\end{array}$ & $\begin{array}{l}\text { Core labs: angiography, duplex } \\
\text { ultrasound, X-ray }\end{array}$ & $\begin{array}{l}\text { Core labs: angiography, duplex } \\
\text { ultrasound, X-ray } \\
\text { Independent clinical events } \\
\text { committee }\end{array}$ & $\begin{array}{l}\text { Independent clinical events } \\
\text { committee }\end{array}$ \\
\hline Primary endpoints & $\begin{array}{l}\text { Objective performance goal efficacy } \\
\text { and safety targets }\end{array}$ & $\begin{array}{l}\text { Objective performance goal efficacy } \\
\text { and safety targets }\end{array}$ & $\begin{array}{l}\text { Efficacy: Freedom from CDTLR at } \\
12 \text { months } \\
\text { Safety: Composite of MAE at } 30 \\
\text { days }\end{array}$ \\
\hline Secondary endpoints & $\begin{array}{l}\text { Primary patency } \\
\text { CDTLR } \\
\text { Changes in Rutherford classification } \\
\text { Changes in ABI measurements }\end{array}$ & $\begin{array}{l}\text { Primary patency } \\
\text { CDTLR } \\
\text { Changes in Rutherford classification } \\
\text { Changes in ABI measurements } \\
\text { Changes in walking impairment } \\
\text { questionnaire }\end{array}$ & $\begin{array}{l}\text { Primary patency } \\
\text { Changes in Rutherford classification } \\
\text { Changes in ABI measurements }\end{array}$ \\
\hline Subgroup analyses & $\begin{array}{l}\text { Post hoc geometric analyses } \\
\text { Presence and quantification of stented } \\
\text { segment curvature based on geometric } \\
\text { analysis of extended-leg and bent-knee } \\
\text { X-ray measurements } \\
\text { Presence and quantification of shear } \\
\text { stress based on computational fluid } \\
\text { dynamic modeling of duplex } \\
\text { ultrasound data and bent-knee X-ray } \\
\text { measurements }\end{array}$ & $\begin{array}{l}\text { Presence and quantification of shear } \\
\text { stress based on computational fluid } \\
\text { dynamic modeling of duplex } \\
\text { ultrasound data and bent-knee } \\
\text { X-ray measurements }\end{array}$ & $\begin{array}{l}\text { Adjunctive stenting with DCBs } \\
(n=50) \\
\text { Popliteal lesions } \\
\text { Calcified lesions }\end{array}$ \\
\hline Inclusion criteria & $\begin{array}{l}\text { Patients with Rutherford category } 1 \text { to } \\
4 \text { with symptoms considered due to } \\
\text { SFA disease } \\
\text { Lesion length: } 4 \mathrm{~cm} \text { to } 10 \mathrm{~cm} \text {, capable } \\
\text { of being treated by a single stent }\end{array}$ & $\begin{array}{l}\text { Patients with Rutherford category } 2 \\
\text { to } 4 \text { due to PAD } \\
\text { Lesion length: } 4 \mathrm{~cm} \text { to } 14 \mathrm{~cm} \text { capable } \\
\text { of being treated by a single stent or } \\
\text { by multiple stents }\end{array}$ & $\begin{array}{l}\text { Patients with documented PAD } \\
\text { who receive the helical centerline } \\
\text { stent in accordance with the IFU }\end{array}$ \\
\hline Exclusion criteria & $\begin{array}{l}\text { Previous interventions at target site } \\
\text { within } 6 \text { months } \\
\text { Previous stent placement in target limb }\end{array}$ & $\begin{array}{l}\text { Target lesion(s) requires } \\
\text { percutaneous interventional } \\
\text { treatment, beyond standard balloon } \\
\text { angioplasty alone, prior to } \\
\text { placement of the study stent }\end{array}$ & $\begin{array}{l}\text { Patients whose lesions that cannot } \\
\text { be crossed with a wire and/or } \\
\text { balloon catheter and cannot be } \\
\text { dilated sufficiently to allow passage } \\
\text { of the delivery system }\end{array}$ \\
\hline Follow-up & 24 months & 36 months & 36 months \\
\hline $\begin{array}{l}\text { Status } \\
\text { S }\end{array}$ & Published results (Zeller et al. [25]) & 12-month results expected in 2018 & 12-month results expected in 2018 \\
\hline
\end{tabular}

it is moderately to severely calcified. The helical centerline curvature of the stent is stored within the shape memory of the nitinol alloy. Patterns of short and long connectors between the strut crowns support the helical geometry and flexibility of the stent. The device design includes transition zones consisting of the last three crowns at each end of the stent. In these transition zones, crowns are increased in length, to reduce the outward radial force of the end of the stent on the vessel wall and to avoid any flow disturbances that might arise due to a step-change between the stent and proximal or distal vessel segments. To avoid the edge restenosis that may be caused by disturbed flow patterns at junctures between stents and normal vessel segments [33], the ends of the helical centerline stent are formed to be collinear with the normal vessel to ensure optimal blood flow into and from the stent. 


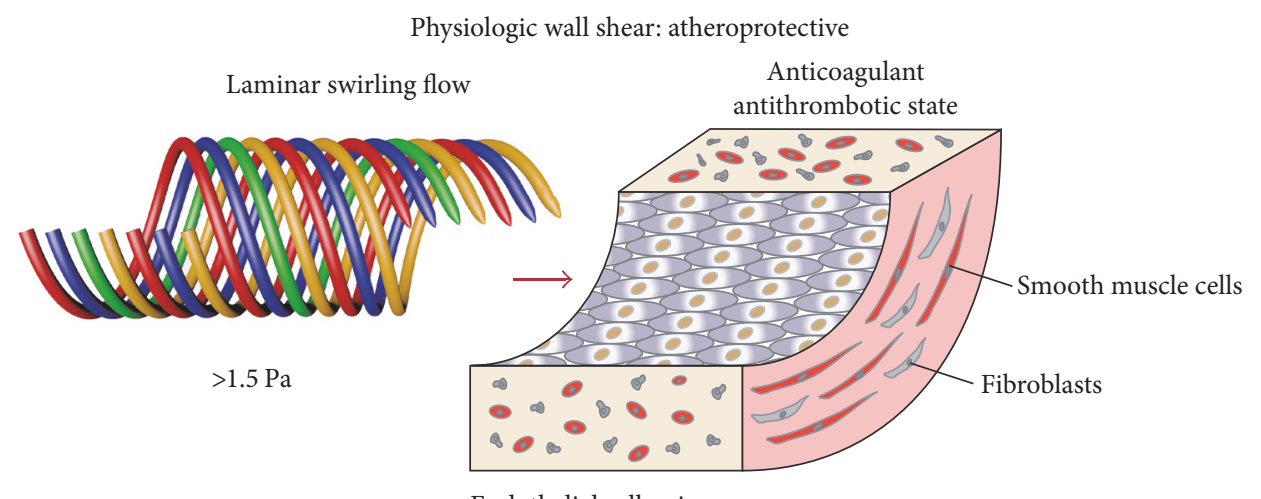

Endothelial cell quiescence

Paracrine quiescence

High antioxidant activity

(a)

Low wall shear: proatherogenic/prorestenotic

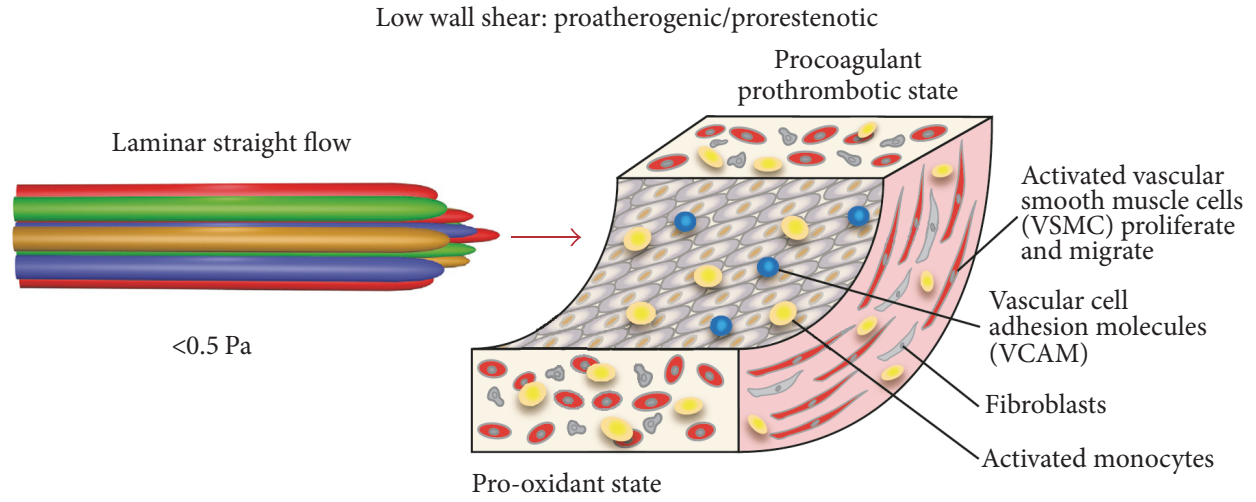

Paracrine proliferative state

(b)

Figure 2: A model of atherogenesis/restenosis, showing differences caused by physiologic arterial wall shear (a) and low (proatherogenic/prorestenotic) wall shear (b) in upregulation of endothelial-cell genes and proteins that are atheroprotective/antirestenotic or atherogenic/restenotic. Adapted from Malek et al. with permission [30].

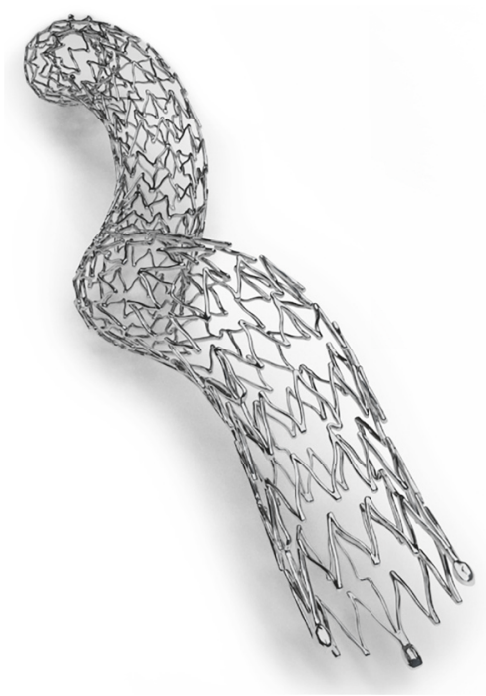

Figure 3: The BioMimics 3D stent (Veryan Medical, Horsham, United Kingdom).
The BioMimics 3D self-expanding helical centerline stent received Conformité Européene (CE) marking in November 2012. The stent is indicated to improve luminal diameter in the treatment of symptomatic de novo and restenotic lesions up to $140 \mathrm{~mm}$ in length in native superficial femoral and/or proximal popliteal arteries with reference vessel diameters ranging from $3.5 \mathrm{~mm}$ to $6.0 \mathrm{~mm}$.

\section{Evaluations to Date of the Helical Centerline Stent}

3.1. Animal Study in Porcine Common Carotid Arteries. In a preclinical study assessing the effect of the helical centerline stent on the development of NIH, the device was compared with a control straight nitinol stent in one or the other of the common carotid arteries of 10 healthy pigs [28]. Digital subtraction angiography, using a thin filament of contrast, was performed immediately after stent deployment and indicated the presence of a swirling blood flow pattern in the helical stent but not in the control straight stent. 

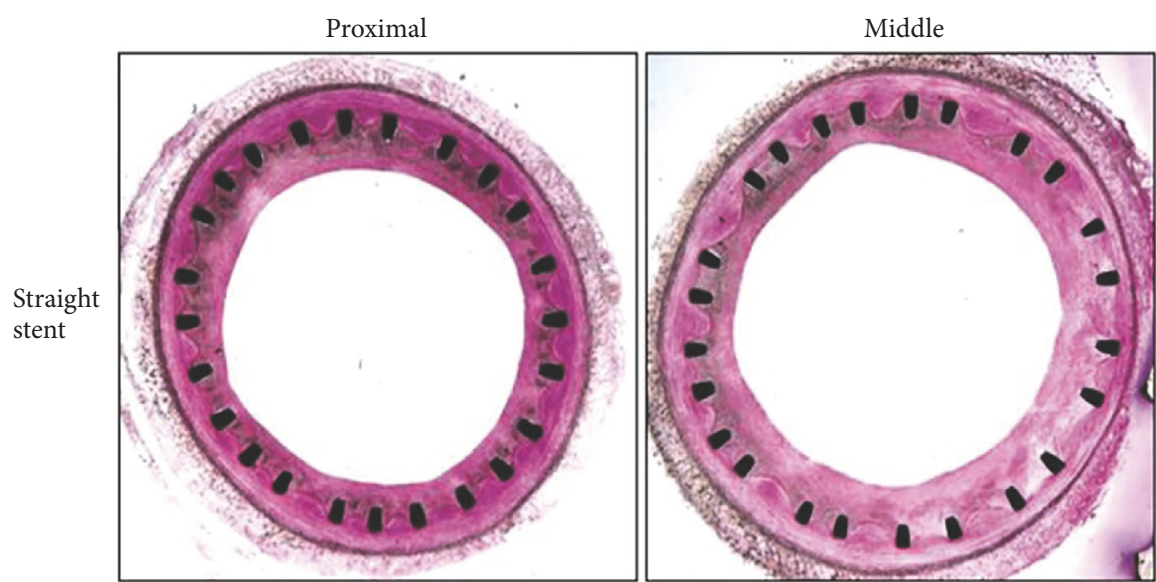

(a)
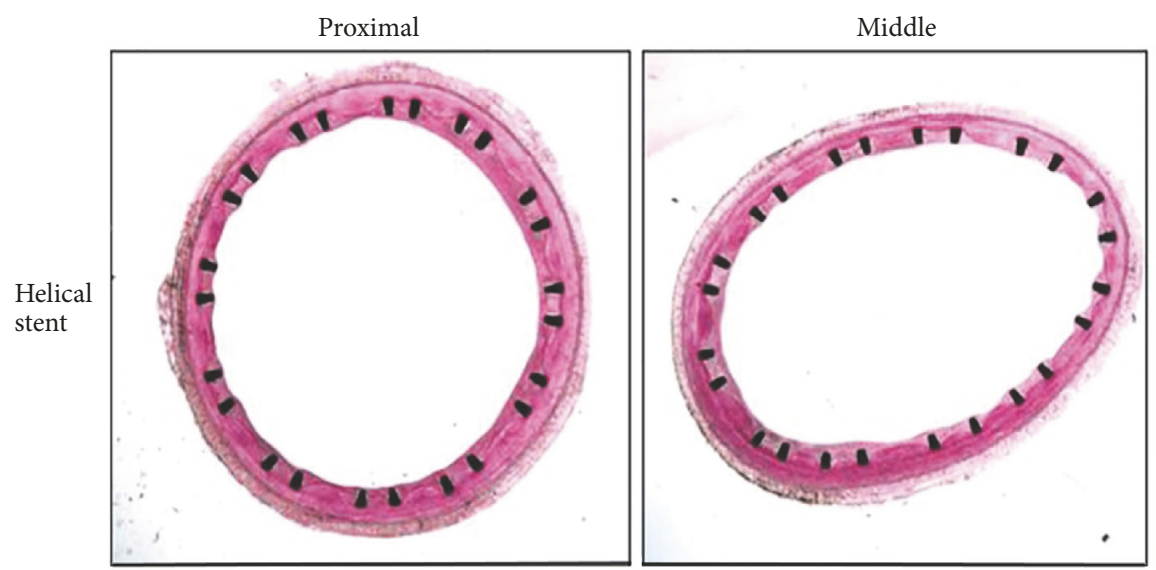

(b)
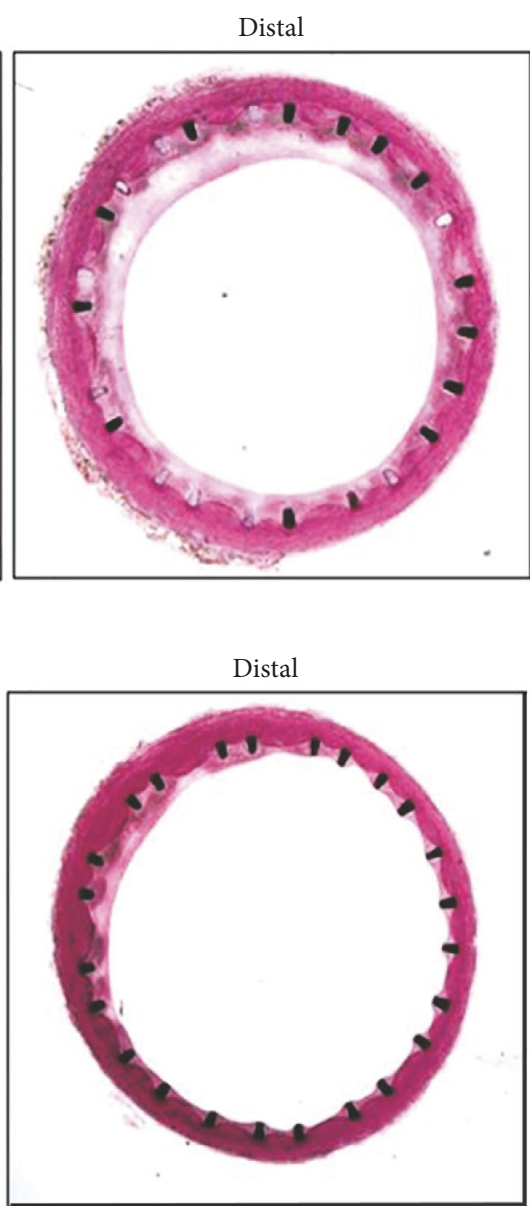

FIGURE 4: Transverse sections of porcine common carotid arteries treated with (a) a control straight stent and (b) the helical centerline stent at 1 month after stent deployment. From Caro et al. with permission [28].

Transverse Doppler ultrasound, using a technique described by How et al. [34], also demonstrated the presence of swirling flow in the helical stent. At animal sacrifice 1 month after deployment, histology revealed significantly less NIH in vessels implanted with the helical centerline stent than in vessels implanted with the control straight stent $(p<0.01)$. Mean neointimal thickness was reduced by an average of $45 \%$ in vessels implanted with the helical centerline stent compared with vessels implanted with the control straight stent $\left(0.2 \pm 0.09 \mathrm{~mm}^{2}\right.$ versus $\left.0.37 \pm 0.08 \mathrm{~mm}^{2}, p<0.05\right)$. Luminal cross-sectional area was significantly greater with the helical centerline stent than with the control straight stent, as can be seen in Figure 4.

Both stents used in this animal study changed the morphology of the common carotid arteries of healthy pigs. However, by imparting a helical curvature to the treated segment, the helical centerline stent distinctly generated a swirling flow of blood. The geometrical and flow changes persisted up to the time of sacrifice at 4 weeks [28]. Swirling flow, as discussed, is associated with a high level of wall shear, which has been found to limit NIH activation and appears to be responsible for the improved histological outcomes with the helical centerline stent in this study [35].

\subsection{The Randomized Controlled MIMICS Trial} (NCT02163863). In the prospective, multicenter, controlled MIMICS trial, 76 patients with TASC (Trans-Atlantic InterSocietal Consensus) II A and B SFA lesions were randomized $2: 1$ to receive the helical centerline stent $(n=50)$ or a control straight nitinol stent (LifeStent, CR Bard, Phoenix, AZ) $(n=26)$. One patient receiving the helical centerline stent had a TASC II C lesion. Ultrasound, angiography, and $\mathrm{X}$-ray imaging review were performed by an independent core laboratory. Eight centers participated in the study, and follow-up continued for 24 months. The primary safety endpoint-freedom from a composite of all-cause mortality, index limb amputation, and TLR through 30 days-was based on an objective performance goal (OPG) of $88 \%$ set by the VIVA Physicians [15]. The primary efficacy endpoint, 6-month freedom from clinically driven TLR (CDTLR), was based on an OPG of $67 \%$ deriving from a review of recent literature [36-38]. The secondary endpoint of 


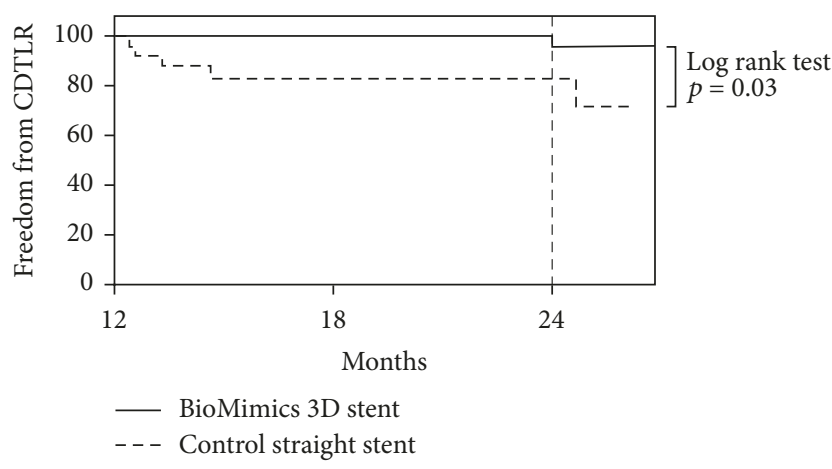

FIGURE 5: Kaplan-Meier curves of freedom from clinically driven target lesion revascularization (CDTLR) after independent event adjudication for the helical centerline stent versus the control straight stent for the landmark period between 12 and 24 months in the MIMICS clinical trial.

primary patency was defined as freedom from $>50 \%$ stenosis identified by formal angiography or duplex ultrasound. Loss of primary stent patency in a treated vessel segment was defined as an increase in the peak systolic velocity ratio of $>2.0$ or the occurrence of CDTLR.

Zeller et al. have published clinical results for the MIMICS trial out to 24 months [25]. The helical centerline stent achieved the primary efficacy and safety endpoints. At 1 year, the Kaplan-Meier survival estimate for primary patency was $80 \%$ for patients who received the helical centerline stent versus $71 \%$ for patients who received the control straight stent. At 2 years, the survival estimate for primary patency was $72 \%$ versus $55 \%$, respectively, a statistically significant primary patency advantage for the helical centerline stent $(\log$-rank $p=0.05)$.

At 1 year, the Kaplan-Meier survival estimate for freedom from CDTLR was $91 \%$ for patients who received the helical centerline stent versus $92 \%$ for patients who received the control straight stent. At 2 years, notably, freedom from CDTLR remained $91 \%$ for patients who received the helical centerline stent while being reduced to $76 \%$ for patients who received the control straight stent. Due to the relatively small sample size, the difference in freedom from CDTLR at 2 years was not statistically significant (log-rank test $p=0.14)$. However, for the period between 12 and 24 months for patients implanted with the helical centerline stent, there were no additional cases of CDTLR, whereas, for patients implanted with the control straight stent, there was a threefold increase in the cases of CDTLR (from $8 \%$ at 12 months to $24 \%$ at 24 months). For this landmark analysis of the period from 12 months to 24 months, there was a statistically significant advantage for the helical centerline stent in freedom from CDTLR (log-rank test $p=0.03$ ) (Figure 5).

A post hoc analysis of the MIMICS trial data was conducted to support understanding of how the BioMimics 3D stent performed with regard to a range of patient risk factors, including grade of calcification, percentage of occlusion, number of patent run-off vessels, diabetes status, and lesion length ( $80 \mathrm{~mm}$ cut-off). For patients implanted with the helical centerline stent, the analysis indicated that there was no significant difference in 24-month primary patency related to any of these variables. There was no reduction in the level of curvature in the implanted stent when patients with no calcification or mild calcification were compared versus those with moderate or severe calcification $(p=0.14)$. Comparing patients with lesion length greater than or less than $80 \mathrm{~mm}$, there was no significant difference in 24-month primary patency for the helical centerline stent $(73 \%$ versus $72 \%, p=0.98$ ), while the patency difference was significant for the control straight stent $(p=0.03)$.

3.3. Helical Curvature, Swirling Flow, and Wall Shear in the MIMCS Trial. Two post hoc geometric analyses, based on $\mathrm{X}$-ray data obtained from all patients in the MIMICS trial, have explored whether the helical centerline stent was able to maintain a higher degree of helical arterial curvature than the control straight stent and then whether stent-modified vessel geometry was associated with a higher level of wall shear due to swirling flow.

During the MIMICS trial, all patients underwent extended-leg and bent-knee X-rays in both anterior/posterior and lateral projections at 1, 6,12, and 24 months. The original purpose of such detailed X-ray evaluation was to inspect devices for evidence of stent fracture (there were no fractures), but the imaging data also provided a basis for computing the three-dimensional helical curvature of each implanted stent. The stent centerline curvature was calculated using a previously described method [39]. Coordinate points on the centerline of the stented vessel were extracted at regular intervals to form the basis for the centerline curvature calculations, which were performed using a MATLAB program (Mathworks, Natick, MA).

The analysis revealed that significantly more stent centerline curvature was present in the extended-leg and bent-knee positions of patients who received the helical centerline stent compared with patients who received the control straight stent. For the extended-leg position, the mean stent centerline curvature was $0.0167 \pm 0.007 \mathrm{~mm}^{-1}$ with the helical centerline stent versus $0.0133 \pm 0.004 \mathrm{~mm}^{-1}$ with the control straight stent $(p=0.019)$. For the bent-knee position, the mean stent curvature was $0.0198 \pm 0.008 \mathrm{~mm}^{-1}$ with the helical centerline stent versus $0.0152 \pm 0.006 \mathrm{~mm}^{-1}$ with the control straight stent $(p=0.018)$. These results in patients from the MIMICS trial confirmed the previous observation from the porcine carotid artery study-that the helical centerline stent imparts a higher level of nonplanar arterial curvature than does a control straight stent, which may be flexible but is not capable of imparting curvature that is not already present in the vessel. Also corroborating and extending the findings of the animal study, qualitative analysis of X-ray data over multiple follow-up visits demonstrated that the helical centerline of the BioMimics 3D stent was maintained over time (Figure 6), providing the basis-through the phenomena of swirling flow and high wall shear-for the significant patency and revascularization outcomes through 24 months in the trial. 


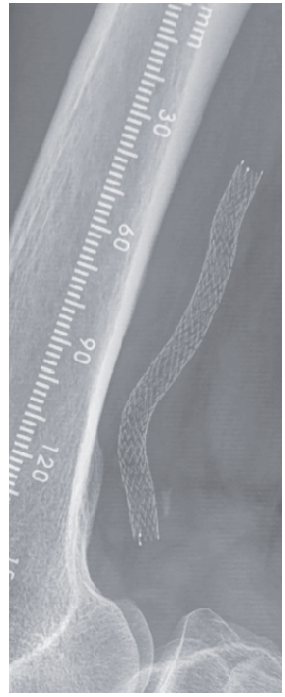

(a)

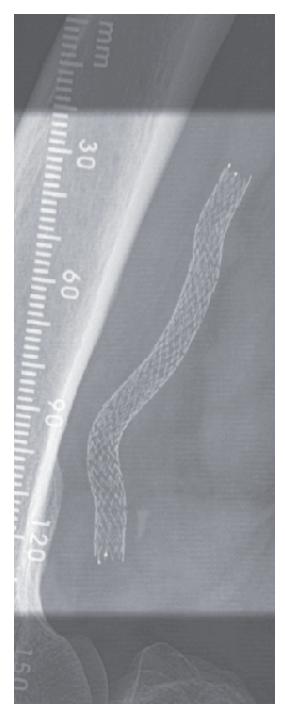

(b)
FIGURE 6: X-ray images of a BioMimics 3D stent in a femoropopliteal location in a patient with knee bent at 90 degrees, at 1 month (a) and 6 months (b) after implantation, showing sustained stent curvature over time.

The reconstructed stent geometry data for the bentknee position were then used for a computational fluid dynamics analysis of the flow characteristics within the lesion segments in order to calculate average wall shear. In this analysis, three-dimensional flow fields were predicted for each stented vessel segment based on patient-specific duplex ultrasound recordings of physiological flow rates, and an engineering simulation suite (ANSYS 14.0, Ansys, Pittsburgh, PA) computed Navier-Stokes equations. In patients treated in the MIMICS trial, the average wall shear as calculated in this analysis was significantly higher within the helical centerline stent than with the control straight stent $(1.13 \pm 0.13$ Pa versus $1.06 \pm 0.12 \mathrm{~Pa}, p=0.05)$.

\section{Conclusion}

Arterial geometry is commonly helical, causing the blood to adopt a laminar swirling flow pattern that increases mixing within the blood and elevates the wall shear on endothelial cells, in turn promoting the diffusion of oxygen to the arterial wall and protecting against the development of atherosclerosis and restenosis [28, 29]. Due to its length and relative straightness, the SFA is exposed to low wall shear under resting conditions and is thus predisposed to atherosclerotic disease and to a confounding of the healing process after the injury associated with endovascular intervention. Implantation into the SFA of a stent with a straight cylindrical configuration will act to further straighten the vessel, rendering it inflexible and potentially jeopardizing the atheroprotective swirling blood flow commonly imparted by the natural helical arterial geometry and leading to activation of the NIH cascade. The BioMimics 3D helical centerline stent was designed to impart a helical shape to vessel morphology and thereby induce a laminar swirling flow that will promote antirestenotic and atheroprotective wall shear. In the MIMICS trial, patients with occlusive SFA lesions were randomized to treatment with either the helical centerline stent or a control straight stent. At 24 months, there was a significant improvement in primary patency for the helical stent compared with the control straight stent. There were no occurrences of CDTLR in the helical centerline stent arm between 12 and 24 months after implantation [25].

The MIMICS trial confirmed that differences in the design of stents for deployment in the femoropopliteal artery are relevant to clinical outcomes. The geometric analysis of X-ray image data for patients in the MIMICS trial found a significantly higher degree of helical curvature with the BioMimics 3D stent than with the control straight stent in both extended-leg and bent-knee configurations, and the imparted curvature was shown to be maintained over time. In a post hoc analysis of the device performance with regard to a range of patient risk factors, there was no reduction in the level of curvature in the implanted stent when patients with no calcification or mild calcification were compared versus those with moderate or severe calcification. The computational fluid dynamics modeling of duplex ultrasound data and bent-knee X-ray measurements indicated a significantly higher level of wall shear with the helical centerline stent than with the control straight stent. It is reasonable, then, to infer that both the higher helical curvature and the higher wall shear associated with the helical centerline stent limited the volume of intervention-activated NIH in stented segments in comparison with the control straight stent. This conclusion is supported by the histological findings in the preclinical study in porcine common carotid arteries [28]. To the end of limiting the volume of NIH activated after endovascular intervention and hence the rate of restenosis, the strategy of attending to the phenomena of swirling flow and wall shear with implantation of the helical centerline stent represents a promising alternative to deployment of DES and a potential complement to the use of DCBs. It remains for the results of the MIMICS trial to be confirmed in the larger MIMICS-2 prospective registry and the MIMICS-3D real-world registry (Table 1).

The MIMICS-3D real-world registry will include an important subgroup analysis of the performance of the helical centerline stent when deployed as a complementary treatment in procedures involving DCBs. The likelihood of there being an important complementary role for a stent-such as the BioMimics 3D-associated with durable outcomes is supported by the observation that while the pharmacokinetics of antiproliferative agents eluted from DCBs is time limited, loss of patency in the SFA due to restenosis occurs as late as 3 to 4 years after treatment [24]. DCBs alone do not provide the scaffolding afforded by a stent for overcoming the recoil and late negative remodeling that are contributory factors in the loss of patency. Besides providing such scaffolding, the BioMimics 3D has been shown in the MIMICS trial to be more capable than a straight nitinol stent in terms of significantly reducing the need for revascularization over an extended term of follow-up. It is thus anticipated that 
the combination of two different strategies for limiting NIH activation-the balloon delivery of antiproliferative drugs and the promotion of swirling flow leading to elevation of antirestenotic and atheroprotective wall shear-will have a synergistic effect on long-term clinical outcomes.

\section{Conflicts of Interest}

Timothy M. Sullivan, Thomas Zeller, Masato Nakamura, Colin G. Caro, and Michael Lichtenberg have been consultants to and have provided research support for Veryan Medical. Colin G. Caro was a founder of Veryan Medical and has shares in the organization.

\section{Acknowledgments}

This work was funded by Veryan Medical, Horsham, UK. Editorial support services were provided by Galen Press, Inc. (Austerlitz, New York, USA) and were paid for by Veryan Medical.

\section{References}

[1] F. G. R. Fowkes, D. Rudan, I. Rudan et al., "Comparison of global estimates of prevalence and risk factors for peripheral artery disease in 2000 and 2010: A systematic review and analysis," The Lancet, vol. 382, no. 9901, pp. 1329-1440, 2013.

[2] M. D. Gerhard-Herman, H. L. Gornik, C. Barrett et al., "AHA/ACC Guideline on the management of patients with lower extremity peripheral artery disease: A report of the American College of Cardiology/American Heart Association Task Force on Clinical Practice Guidelines," J Am Coll Cardiol, vol. 69, no. 11, pp. e71-e126, 2016.

[3] C. Kasapis and H. S. Gurm, "Current approach to the diagnosis and treatment of femoral-popliteal arterial disease. A systematic review," Current Cardiology Reviews, vol. 5, no. 4, pp. 296-311, 2009.

[4] A. Diamantopoulos and K. Katsanos, "Treating femoropopliteal disease: Established and emerging technologies," Seminars in Interventional Radiology, vol. 31, no. 4, pp. 345-352, 2014.

[5] R. Ní Ghriallais, K. Heraty, B. Smouse, M. Burke, P. Gilson, and M. Bruzzi, "Deformation of the femoropopliteal segment: Effect of stent length, location, flexibility, and curvature," Journal of Endovascular Therapy, vol. 23, no. 6, pp. 907-918, 2016.

[6] J. W. Jukema, J. J. W. Verschuren, T. A. N. Ahmed, and P. H. A. Quax, "Restenosis after PCI. Part 1: Pathophysiology and risk factors," Nature Reviews Cardiology, vol. 9, no. 1, pp. 53-62, 2011.

[7] S. G. Carlier, L. C. A. Van Damme, C. P. Blommerde et al., "Augmentation of wall shear stress inhibits neointimal hyperplasia after stent implantation: Inhibition through reduction of inflammation?" Circulation, vol. 107, no. 21, pp. 2741-2746, 2003.

[8] G. G. Caro, "Discovery of the role of wall shear in atherosclerosis," Arteriosclerosis, Thrombosis, and Vascular Biology, vol. 29, no. 2, pp. 158-161, 2009.

[9] N. B. Wood, S. Z. Zhao, A. Zambanini et al., "Curvature and tortuosity of the superficial femoral artery: A possible risk factor for peripheral arterial disease," Journal of Applied Physiology, vol. 101, no. 5, pp. 1412-1418, 2006.

[10] V. L. Rowe, W. Lee, F. A. Weaver, and D. Etzioni, "Patterns of treatment for peripheral arterial disease in the United States:
1996-2005," Journal of Vascular Surgery, vol. 49, no. 4, pp. 910917, 2009.

[11] L. Norgren, W. R. Hiatt, J. A. Dormandy, M. R. Nehler, K. A. Harris, and F. G. R. Fowkes, "Inter-society consensus for the management of peripheral arterial disease (TASC II)," Journal of Vascular Surgery, vol. 45, no. 1, pp. S5-S67, 2007.

[12] W. P. Robinson III, L. L. Nguyen, R. Bafford, and M. Belkin, "Results of second-time angioplasty and stenting for femoropopliteal occlusive disease and factors affecting outcomes," Journal of Vascular Surgery, vol. 53, no. 3, pp. 651-657, 2011.

[13] B. C. Kearns and S. M. Thomas, "Cost-effectiveness of superficial femoral artery endovascular interventions in the UK and Germany: A modelling study," BMJ Open, vol. 7, no. 1, Article ID e013460, 2017.

[14] E. M. Mahoney, K. Wang, H. H. Keo et al., "Vascular hospitalization rates and costs in patients with peripheral artery disease in the United States," Circulation: Cardiovascular Quality and Outcomes, vol. 3, no. 6, pp. 642-651, 2010.

[15] K. J. Rocha-Singh, M. R. Jaff, T. R. Crabtree, D. A. Bloch, and G. Ansel, "Performance goals and endpoint assessments for clinical trials of femoropopliteal bare nitinol stents in patients with symptomatic peripheral arterial disease," Catheterization and Cardiovascular Interventions, vol. 69, no. 6, pp. 910-919, 2007.

[16] N. Chalmers, P. T. Walker, A.-M. Belli et al., "Randomized trial of the SMART stent versus balloon angioplasty in long superficial femoral artery lesions: The SUPER study," CardioVascular and Interventional Radiology, vol. 36, no. 2, pp. 353-361, 2013.

[17] H. Krankenberg, M. Schlüter, H. J. Steinkamp et al., "Nitinol stent implantation versus percutaneous transluminal angioplasty in superficial femoral artery lesions up to $10 \mathrm{~cm}$ in length: the Femoral Artery Stenting Trial (FAST)," Circulation, vol. 116, no. 3, pp. 285-292, 2007.

[18] K. Katsanos, G. Tepe, D. Tsetis, and F. Fanelli, "Standards of practice for superficial femoral and popliteal artery angioplasty and stenting," CardioVascular and Interventional Radiology, vol. 37, no. 3, pp. 592-603, 2014.

[19] G. Tepe, J. Laird, P. Schneider et al., "Drug-coated balloon versus standard percutaneous transluminal angioplasty for the treatment of superficial femoral and popliteal peripheral artery disease: 12-month results from the IN.PACT SFA randomized trial," Circulation, vol. 131, no. 5, pp. 495-502, 2015.

[20] J. R. Laird, P. A. Schneider, G. Tepe et al., "Durability of treatment effect using a drug-coated balloon for femoropopliteal lesions: 24-month results of IN.PACT SFA," Journal of the American College of Cardiology, vol. 66, no. 21, pp. 2329-2338, 2015.

[21] D. Scheinert, K.-L. Schulte, T. Zeller, J. Lammer, and G. Tepe, "Paclitaxel-releasing balloon in femoropopliteal lesions using a BTHC excipient: Twelve-month results from the BIOLUX P-I randomized trial," Journal of Endovascular Therapy, vol. 22, no. 1, pp. 14-21, 2015.

[22] H. Schroeder, D.-R. Meyer, B. Lux, F. Ruecker, M. Martorana, and S. Duda, "Two-year results of a low-dose drug-coated balloon for revascularization of the femoropopliteal artery: Outcomes from the ILLUMENATE first-in-human study," Catheterization and Cardiovascular Interventions, vol. 86, no. 2, pp. 278-286, 2015.

[23] K. Rosenfield, M. Jaff, and C. White, "Trial of a paclitaxel-coated balloon for femoropopliteal artery disease," Journal of Vascular Surgery, vol. 373, no. 2, pp. 145-153, 2015. 
[24] O. Iida, M. Uematsu, Y. Soga et al., "Timing of the restenosis following nitinol stenting in the superficial femoral artery and the factors associated with early and late restenoses," Catheterization and Cardiovascular Interventions, vol. 78, no. 4, pp. 611-617, 2011.

[25] T. Zeller, P. A. Gaines, G. M. Ansel, and C. G. Caro, "Helical centerline stent improves patency: Two-year results from the randomized mimics trial," Circulation: Cardiovascular Interventions, vol. 9, no. 6, Article ID e002930, 2016.

[26] C. G. Caro, J. M. Fitz-Gerald, and R. C. Schroter, "Arterial wall shear and distribution of early atheroma in man," Nature, vol. 223, no. 5211, pp. 1159-1161, 1969.

[27] C. G. Caro, D. J. Doorly, M. Tarnawski, K. T. Scott, Q. Long, and C. L. Dumoulin, "Non-planar curvature and branching of arteries and non-planar-type flow," Proceedings of the Royal Society A Mathematical, Physical and Engineering Sciences, vol. 452, no. 1944, pp. 185-197, 1996.

[28] C. G. Caro, A. Seneviratne, K. B. Heraty et al., "Intimal hyperplasia following implantation of helical-centreline and straight-centreline stents in common carotid arteries in healthy pigs: Influence of intraluminal flow," Journal of the Royal Society Interface, vol. 10, no. 89, Article ID 20131156, 2013.

[29] G. Coppola and C. Caro, "Oxygen mass transfer in a model three-dimensional artery," Journal of the Royal Society Interface, vol. 5, no. 26, pp. 1067-1075, 2008.

[30] A. M. Malek, S. L. Alper, and S. Izumo, "Hemodynamic shear stress and its role in atherosclerosis," The Journal of the American Medical Association, vol. 282, no. 21, pp. 2035-2042, 1999.

[31] J.-J. Chiu, S. Usami, and S. Chien, "Vascular endothelial responses to altered shear stress: Pathologic implications for atherosclerosis," Annals of Medicine, vol. 41, no. 1, pp. 19-28, 2009.

[32] J.-J. Chiu and S. Chien, "Effects of disturbed flow on vascular endothelium: pathophysiological basis and clinical perspectives," Physiological Reviews, vol. 91, no. 1, pp. 327-387, 2011.

[33] F. J. Arena, "Arterial kink and damage in normal segments of the superficial femoral and popliteal arteries abutting nitinol stents-A common cause of late occlusion and restenosis? A single-center experience," The Journal of Invasive Cardiology, vol. 17, no. 9, pp. 482-486, 2005.

[34] T. V. How, R. K. Fisher, J. A. Brennan, and P. L. Harris, "Swirling flow pattern in a non-planar model of an interposition vein cuff anastomosis," Medical Engineering \& Physics, vol. 28, no. 1, pp. 27-35, 2006.

[35] G. Coppola and C. Caro, "Arterial geometry, flow pattern, wall shear and mass transport: Potential physiological significance," Journal of the Royal Society Interface, vol. 6, no. 35, pp. 519-528, 2009.

[36] G. Tepe, T. Zeller, T. Albrecht et al., "Local delivery of paclitaxel to inhibit restenosis during angioplasty of the leg," The New England Journal of Medicine, vol. 358, no. 7, pp. 689-699, 2008.

[37] M. Werk, S. Langner, B. Reinkensmeier et al., "Inhibition of restenosis in femoropopliteal arteries. Paclitaxel-coated versus uncoated balloon: Femoral paclitaxel randomized pilot trial," Circulation, vol. 118, no. 13, pp. 1358-1365, 2008.

[38] J. R. Laird, B. T. Katzen, D. Scheinert et al., "Nitinol stent implantation vs. balloon angioplasty for lesions in the superficial femoral and proximal popliteal arteries of patients with claudication: Three-year follow-up from the resilient randomized trial," Journal of Endovascular Therapy, vol. 19, no. 1, pp. 1-9, 2012.
[39] P. M. O’Flynn, G. O’Sullivan, and A. S. Pandit, "Methods for three-dimensional geometric characterization of the arterial vasculature," Annals of Biomedical Engineering, vol. 35, no. 8, pp. 1368-1381, 2007. 


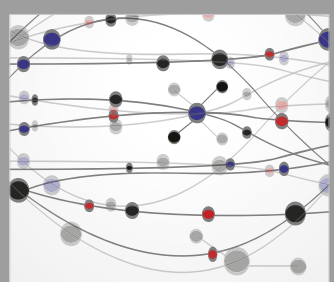

The Scientific World Journal
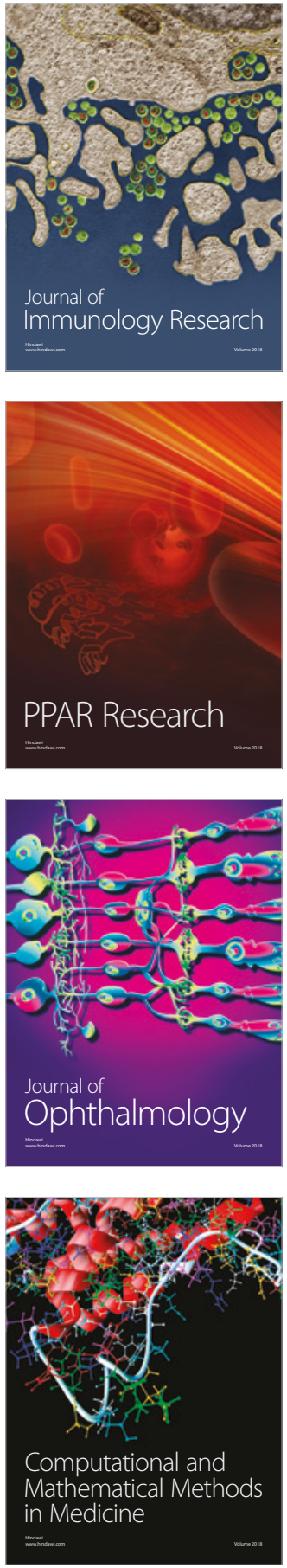

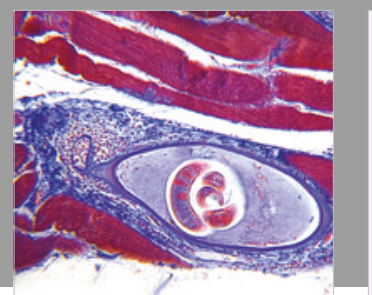

Gastroenterology Research and Practice

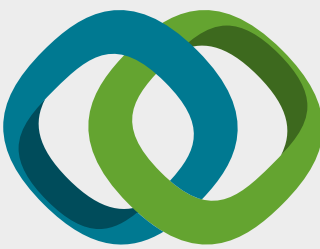

\section{Hindawi}

Submit your manuscripts at

www.hindawi.com
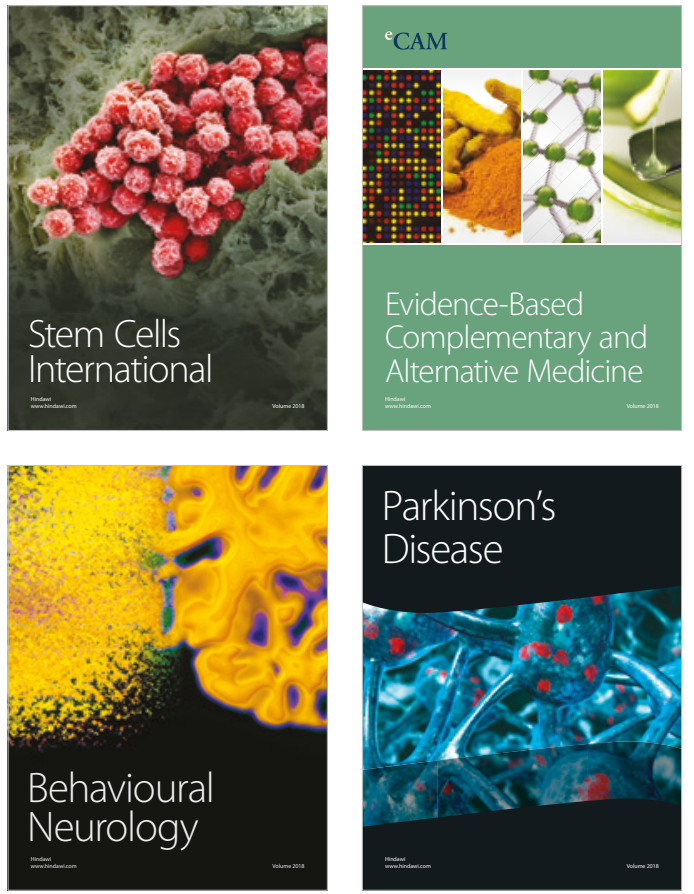

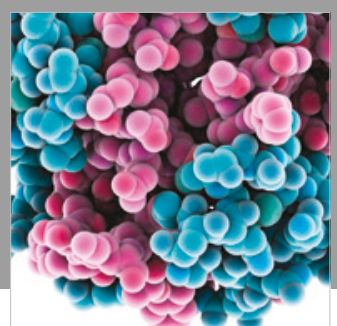

ournal of

Diabetes Research

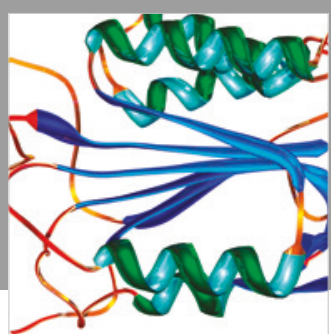

Disease Markers
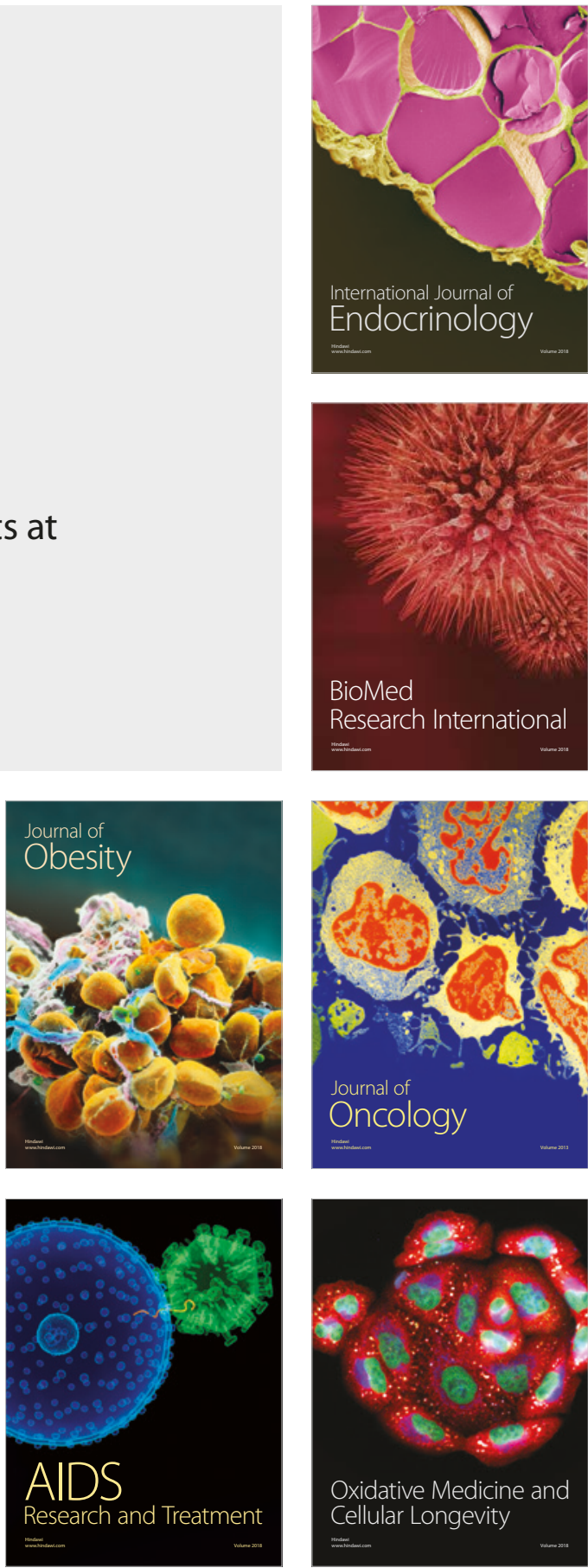